\title{
Resonance Cones in Magnetized Plasma
}

C. Riccardi, M. Salierno, P. Cantu', M. Fontanesi and Th. Pierre*

Dipartimento di Fisica - Universita' degli Studi di Milano, Via Celoria, 16-20133 Milano,

Italy

* Laboratoire de Physique des Milieux Ionisés, C.N.R.S., Université de Nancy I,

B.P. 239, 54506 Vandouvre-les-Nancy cedex, France

\begin{abstract}
An experimental investigation of ion and electron resonance cones have been carried out in the toroidal device Thorello in which a weakly magnetized plasma is produced. As resonance cones propagation depends on foundamental parameters of plasma, we can estimate density, temperature, magnetic field and ion minority concentration. In this way the investigation aims to develop some plasma diagnostics comparable to the other methods.
\end{abstract}

\section{INTRODUCTION}

It is known that the fields of an oscillating point source in a cold magnetized plasma, become singular on a cone surface (called 'resonance cone') whose axis is parallel to the static magnetic field and whose half-angle $\theta$ is determined from plasma parameters.

Fisher and Gould have shown that there are three frequency ranges in which the resonance cone can exists: $\max \left(\Omega_{\mathrm{e}}, \omega_{\mathrm{pe}}\right)<\omega<\omega_{\mathrm{uh}}, \omega_{\mathrm{lh}}<\omega<\min \left(\Omega_{\mathrm{e}}, \omega_{\mathrm{pe}}\right), \omega<\Omega_{\mathrm{i}}$ where $\omega_{\mathrm{uh}}, \omega_{\mathrm{lh}}, \Omega_{\mathrm{c}}$ and $\Omega_{\mathrm{i}}$ are the upper hybrid, lower hybrid, electron cyclotron and ion cyclotron frequencies and $\omega_{\mathrm{pe}}$ is the electron plasma frequency.

The propagation characteristics of resonance cones in magnetized plasma strongly depends on the plasma parameters so that it is possible to use them as diagnostic method.

An accurate analysis of resonance cones has been curried out in the toroidal device Thorello which produces low density magnetized plasma: $\mathrm{n}_{\mathrm{e}}<10^{11} \mathrm{~cm}^{-3}, \mathrm{~T}_{\mathrm{e}}<10 \mathrm{eV}, \mathrm{T}_{\mathrm{j}}<2 \mathrm{eV}, \mathrm{B}<2 \mathrm{KG}$ auss.

In this analysis we excited both ion and electron resonance cones.

The wave potential obtained with a point source is given by [1]:

$$
\Phi(\vec{r}, t)=\frac{e^{-i \omega t}}{2 \pi^{2}} \int d^{3} \vec{k} \frac{e^{i \vec{k} \cdot \vec{r}}}{\left(k_{\perp}^{2} K_{x x}+k_{\|}^{2} K_{z z}\right)}
$$

where $\mathrm{Kxx}$ and $\mathrm{Kzz}$ depend on the plasma species. 
As the antenna is a point source antenna, the wave potential in plasma is given by the integral of several waves with different $k_{\mathrm{ll}}$; it turns out that the wave potential has very high values in corrispondence of some spatial trajectories characterized by the cone angle which is defined by :

$$
\theta=\operatorname{artg} \frac{k_{\|}}{k_{\perp}}
$$

\section{ION CONES}

The ion cones are excited at low frequencies $\left(\omega<\Omega_{i}\right)$ and depend mainly on the ion species; for this reason they can be used for the extimation of the concentration of ion minorities.

The relevant dispersion relation is given by the following formula [2]:

$$
1+\frac{k_{\perp}^{2}}{k^{2}} \sum_{\sigma \neq e} \frac{\omega_{p \sigma}^{2}}{\left(\Omega_{\sigma}^{2}-\omega^{2}\right)}+\frac{k_{\perp}^{4}}{k^{2}} \sum_{\sigma \neq e} \frac{3 \omega_{p \sigma}^{2} \frac{T_{\sigma}}{m_{\sigma}}}{\left(4 \Omega_{\sigma}^{2}-\omega^{2}\right)\left(\omega^{2}-\Omega_{\sigma}^{2}\right)}-\frac{k_{\|}^{2}}{k^{2}} \frac{\omega_{p e}^{2}}{\omega^{2}}=0
$$

where we have considered the presence of a minority of $H_{3}^{+}$ions besides the $H^{+}$ion species; in our plasma a high percentage $(<50 \%)$ of $H_{3}^{+}$ions is produced.

To detect ion cones it has been employed an interferometric system that permit to measure a signal proportional to the electric field of electrostatic wave propagating in the plasma.

The launching system is made by an antennas set, whose signal depends on geometrical characteristics : a slow wave antenna, made by one or more plates aligned along the toroidal coordinate, and a point source antenna. The signal is detected with RF probe. Both the antenna and the probe are located in the equatorial plane. In Fig.1 the amplitude of the signal has been plotted for different frequencies. The experimental estimation of the resonance cone angle is geometrically performed considering the ratio between $\Delta r$ (the radial shift of the potential maximum with respect to the antenna position) and $\Delta \mathrm{z}$ (the distance between the antenna and the receiving probe evaluated along the toroidal coordinate) :

$$
\theta=\arctan (\Delta r / \Delta z)
$$

The estimation of the minority ion concentration is performed by fitting the experimental curve of $\theta$ vs. $\omega / \Omega_{i}$ with a theoretical one achievable through eq. 3 , where the definition of $\theta$ has to be substituted.

Fig.2 is related to an experimental situation for which we measured following concentration of $H_{3}^{+}$ ions in respect to the electron density : $n_{H_{3}^{+}}=50 \%$. The sensitivity of the ion concentration is about $5 \%$, as shown in same graphic. This diagnostic method can be also used in magnetized plasmas of higher density (up to $10^{12} \mathrm{~cm}^{-3}$ ). If the source is broad only some specific waves modes can be excited. In this case the so called resonance cone becomes spatially broadened so that its envelope presents several wavelengths. The ion temperature effect is similar to the one in the previsious case in so far we can observe how the wavelengths change with the ion temperature. 


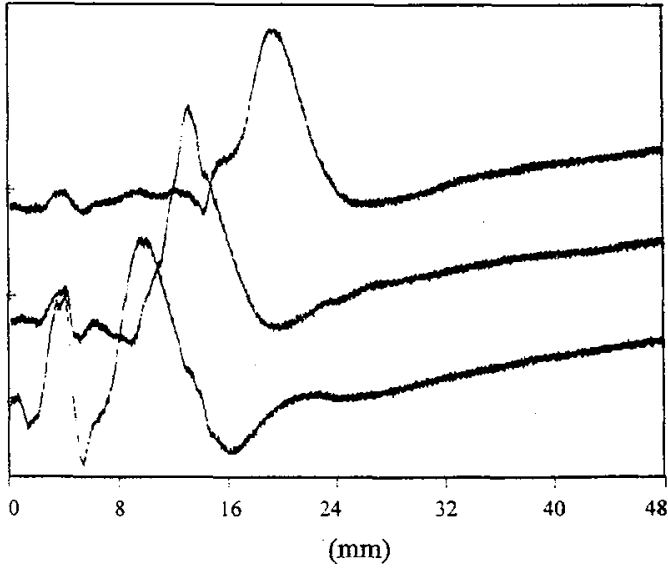

Fig. I Experimental interferograms (in a.u.) performed at the frequencies (from the top) :3 MHz, 2.7 MHz, 2.3 $M \mathrm{~Hz}$, with $B_{\text {toroidal }}=2.15 \mathrm{KG}$. The radial shift of the resonance cone can be seen.

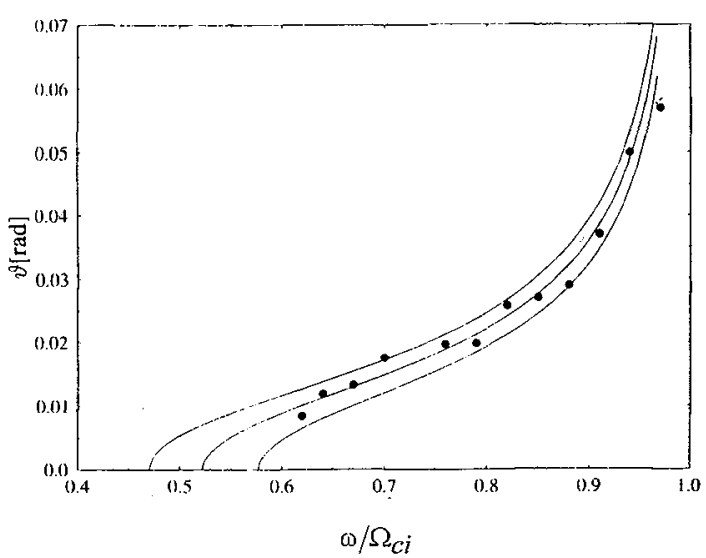

Fig.2 Ion cones propagation angle vs launching frequency : comparison between experimental points and thoeretical curves plotted for the following $H^{+}$concentration: $62 \%, 52 \%$ and, $42 \%$.

\section{ELECTRON CONES}

The electron resonance cones depend on the electron density, on the electron temperature and on the magnetic field intensity; they can be excited in following frequency ranges $0<\omega<\min \left(\omega_{p e}, \Omega_{e}\right)$, $\max \left(\omega_{p e}, \Omega_{e}\right)<\omega<\omega_{k b}$, where $\omega_{x b}$ is the upper hybrid frequency $\omega_{u h}=\sqrt{\left(\omega_{p e}^{2}+\omega_{\alpha}^{2}\right)}$.

The components of the dielettric tensor are : $K_{x x}=1+\frac{\omega_{p_{e}}^{2}}{\Omega_{e}^{2}-\omega^{2}} \quad ; \quad K_{z z}=\left(1-\frac{\omega_{p_{e}}^{2}}{\omega^{2}}-\frac{3}{2} \frac{k_{\|_{e}}^{2} v_{t_{e}}^{2} \omega_{p_{e}}^{2}}{\omega^{4}}\right)$ In a cold plasma the resonance cone angle is given by :

$$
\sin ^{2} \theta=\frac{\omega^{2}\left(\omega_{p_{e}}^{2}+\Omega_{e}^{2}-\omega^{2}\right)}{\omega_{p e}^{2} \Omega_{e}^{2}}
$$

There are two resonance frequencies for which the wave signal can achieve its maximum value

To analyse the resonance cones we use a double r.f. probe, composed by two pins in which one is the transmitter, fed with sinusoidal signals of the same amplitude but different frequencies, the other is the receiver located far away from the transmitter of a few tenth millimeters .

The pin length is along the radial coordinate. The probe can move radially and the plane of the probe (which contains the two pins) can rotate in a plane perpendicular to the magnetic field.

To measure the electron density avoiding electron temperature effects, we can rotate the probe perpendicularly to the toroidal magnetic field in order to $\operatorname{set} \theta=90^{\circ}$ : the resonance frequency corresponds to the electron plasma frequency and depends only on the density: $f_{r e s}=f_{p e} \propto\left(n_{e}\right)^{1 / 2}$. 
The plot of the signal amplitude vs the frequency, given in Fig.3, shows the typical shape of resonance cone: the electron density is estimated measuring the plasma frequency.

At differents angles the resonance frequencies depend on the magnetic field intensity and on the electron temperature. This wave based diagnostic method has been compared with the Langmuir probe method: the density profile obtained through the first method lies between the two profiles obtained by the latter ones. The precision of the method mainly depends on the presence of density fluctuations that can cause an high indetermination of the resonance peak.

In the same perpendicular position $\theta=90^{\circ}$ the second resonance frequency corresponds to the electron cyclotron frequency $f_{r e s}=f_{c e} \propto B$ so we can directly measure the magnetic field intensity. As our signal generator can generate frequency below $1 \mathrm{GHz}$, to performed this diagnostic we decrease the magnetic field intensity below 360 Gauss. The resonance cone is shown if Fig.4, from that we obtain the following intensity: 260 Gauss, according to that obtained with an Hall probe. The magnetic field estimation with resonance cones has been analysed in a large range of different magnetic field intensities giving a good accuracy.

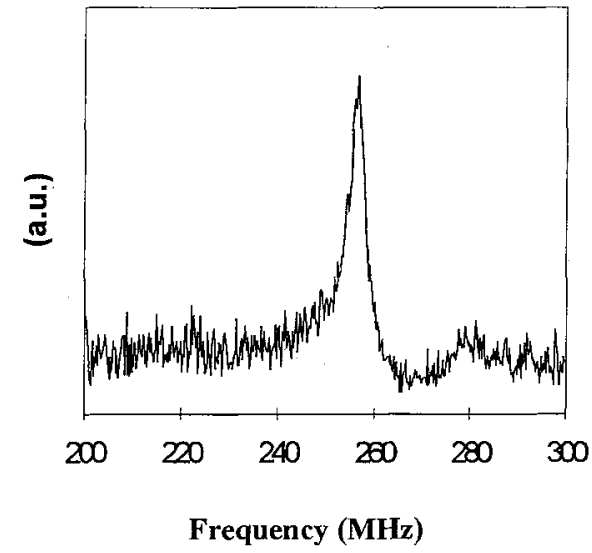

Fig.3 Resonance cone for the electron density estimation : $n_{e}=8.1 \times 10^{8} \mathrm{~cm}^{-3}$

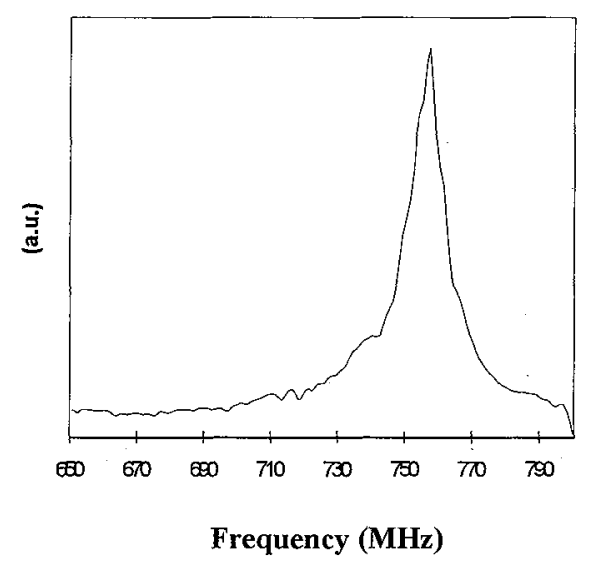

Fig. 4 Resonance cone for the magnetic field estimation : $f_{\text {res }}=f_{c e}=\Omega_{e} / 2 \pi, B=260 \mathrm{G}$

\section{REFERENCE}

[1] T. H. Stix, The Theory of Plasma Waves, McGraw-Hill (1962)

[2] M. Ono, K. L. Wong, G. A. Wurden, Phys. Fluids, 26, 1, (1983)

[3] P. K. Fisher, R. W. Gould, Phys. Fluids, 14, 857, (1971) 